\title{
The rapid kinetics of optimal treatment with subcutaneous methotrexate in early inflammatory arthritis: an observational study
}

Anna O'Connor ${ }^{1}$, Carter Thorne ${ }^{2}$, Hyeon Kang ${ }^{3}$, Diane $\operatorname{Tin}^{2}$ and Janet E. Pope ${ }^{1,3,4^{*}}$

\begin{abstract}
Background: Methotrexate (MTX) is standard treatment for RA. Absorption is better in subcutaneous MTX (scMTX), which may impact speed of onset. In RA, earlier time to remission improves long-term results. Our objectives were to determine rapidity of response of subcutaneous methotrexate in early rheumatoid arthritis.
\end{abstract}

Methods: The change in several disease activity measures (including DAS28) from 0 to 6 weeks (early period) and 6 to 12 weeks (late period) was compared. The proportion achieving DAS28/CDAI/SDAl remission and/or low disease activity state was also compared.

Results: One hundred three patients were included from a single site between 2008 and 2014. All received MTX (98.0 \% scMTX, 98 \% 25 mg/week). There were no dropouts. There was a significantly greater early change in DAS28 ( -1.9 vs. $-0.2, p<0.00)$; this effect was seen for several outcome measures. By 6 weeks, $59 \%$ had achieved either DAS28 remission or low disease activity state, with $74 \%$ achieving either state by 12 weeks.

There were a larger proportion of patients achieving CDAI and DAS28 remission in the early versus late period $(p<0.0002$ for both). There was significant improvement when using combination MTX and HCQ, however sample size was small $(n=9)$. The use of intra-articular steroids with MTX yielded the most disease measures that demonstrated early significant improvement.

Conclusion: Subcutaneous MTX is rapid, as the change in many disease activity scores was significantly greater between 0-6 weeks compared to 6-12 weeks. Combination MTX + HCQ gave added value, although generalizability is limited by combination cohort sample size. Intra-articular steroid injections may contribute to the early effect.

Keywords: ERA (early RA), Methotrexate, Optimal treatment strategy, Subcutaneous (sc)

Abbreviations: ACR, American College of Rheumatology; ANOVA, Analysis of variance; $C A T C H$, Canadian early arthritis cohort; CDAl, Clinical disease activity index; DAS28, Disease activity score (28 joint count); DMARD, Disease modifying anti-rheumatic drug; EIA, Early inflammatory arthritis; HCQ, Hydroxychloroquine; MTX, Methotexate; RA, Rheumatoid arthritis; sc, Subcutaneous; scMTX, Subcutaneous methotrexate; SDAl, Simplified disease activity index

\footnotetext{
* Correspondence: janet.pope@sjhc.london.on.ca

${ }^{1}$ Schulich School of Medicine \& Dentistry, University of Western Ontario, 268 Grosvenor St, London, ON N6A 4V2, Canada

${ }^{3}$ University of Toronto, Toronto, ON, Canada

Full list of author information is available at the end of the article
} 


\section{Background}

Rheumatoid arthritis (RA) is a chronic autoimmune inflammatory disease characterized by joint swelling, pain that can lead to joint damage, functional impairment, disability, deterioration of quality of life, and premature mortality [1-3]. Initiating treatment for RA early in the disease process can lead to improved long-term outcomes, suggesting that there is an optimal window of opportunity for achieving treatment targets [2, 4-6].

Despite the existence of newer anti-rheumatic drugs (disease modifying anti-rheumatic drugs; DMARDs), methotrexate (MTX) remains the most widely used DMARD because it is effective, has an acceptable toxicity profile, and inexpensive [7-11]. Dosing and route of MTX administration have evolved. Early trials started with low doses (5-7.5 mg/week); however, modern practice typically involves starting at or rapidly escalating to higher doses (15-25 mg/week) for optimal outcomes [12-15]. Route of MTX delivery may also impact disease outcomes; bioavailability is higher when given subcutaneously (sc), possibly relating to limitations in gastrointestinal tract absorption and patient adherence secondary to GI side effects $[12,13]$. A previous study of oral and sc MTX in patients with RA suggested that oral administration was less effective [12]. A separate observational study demonstrated a significant association between initial sc administration of MTX and treatment continuation over the first year compared to oral MTX in patients with early RA [13].

The kinetics of optimal initial dosing with subcutaneous methotrexate are unclear. Methotrexate has traditionally been considered slow acting, especially in comparison to newer biologic therapies, with recommendations to reassess clinical response to MTX at 12 weeks [7]. However, this has not been our clinical impression with parenteral MTX. The objective of this study was to determine the kinetics of optimizing initial dosing with sc MTX in patients with early rheumatoid arthritis, comparing outcomes at 6 and 12 weeks after initiation of methotrexate with or without other treatment such as intra-articular steroids.

\section{Methods}

\section{Study design}

Data were collected retrospectively from a single site inception cohort of patients enrolled into the Canadian Early Arthritis Cohort (CATCH) study. CATCH is an observational, prospective "real world" cohort of patients with early inflammatory arthritis (EIA) recruiting at 15 sites since July 2007. Inclusion criteria were: age $>16$ years, between 6 weeks and 12 months of persistent synovitis at time of entry, and $\geq 2$ swollen joints or 1 swollen metacarpophalangeal or proximal interphalangeal joint and $\geq 1$ of the following were required: positive rheumatoid factor (RF), positive anti-cyclic citrullinated peptide (anti-CCP), morning stiffness $>45 \mathrm{~min}$, response to nonsteroidal anti-inflammatory drugs, or painful metatarsophalangeal squeeze test. Outcomes are collected at each visit including global assessments, joint counts, and inflammatory markers. Treatment was left to the discretion of the treating physician and included: monotherapy or combination DMARD therapy, steroids, NSAIDs and biologics. $\mathrm{CATCH}$ patients enrolled at a single site (Southlake Regional Health Centre) were included if they: attended an additional "early follow-up" appointment $6 \pm 2$ weeks from baseline, attended a 12-week follow up appointment, were prescribed methotrexate within 3 months from their baseline appointment, and were on methotrexate at least 1 month between baseline and the $6 \pm 2$ week follow-up appointment. At early follow-up, the standard $\mathrm{CATCH}$ protocol was used to evaluate patients. Data from early follow-up were collected from paper and/or electronic records as this visit was not part of the standard $\mathrm{CATCH}$ protocol. One individual (HK) was responsible for data collection and coding.

Disease measures assessed at baseline, 6 and 12 weeks included: swollen joint count (SJC), 28-tender joint count (TJC28), 28-swollen joint count (SJC28), 28-joint disease activity score (DAS28ESR, DAS28CRP), sleep, pain, fatigue, physician global assessment, patient global assessment, health assessment questionnaire (HAQ), ESR, and CRP. Other composite scores were calculated, including the clinical disease activity index (CDAI) and simplified disease activity index (SDAI). Data regarding tolerability to methotrexate were also collected, including incidence of headache, nausea, and gastrointestinal side effects.

\section{Statistical methods}

A repeated measures ANOVA was performed to compare each disease activity score at baseline, 6 and 12 weeks. A repeated measures ANOVA was also performed on the calculated mean difference in disease activity scores between baseline and 6 weeks, 6 and 12 weeks, and baseline and 12 weeks. A paired $t$-test was performed to compare the mean difference in disease activity scores between early (baseline to 6 weeks) and late (6-12 weeks) groups.

Subgroup analyses assessed the effect of confounding concomitant steroid use and combination therapy with HCQ. Patients were classified according to route of steroid use: intra-articular, oral, intra-muscular, combination, and no steroid. A repeated-measures ANOVA compared raw disease activity scores and the mean difference between time points. Post hoc analysis compared mean scores between early (baseline to 6 weeks) and late (6-12 weeks) groups across routes of steroid use. A similar analysis was performed to compare scores in patients receiving monotherapy versus combination therapy with HCQ. 


\section{Results}

\section{Patient population}

A total of 103 of $209 \mathrm{CATCH}$ patients enrolled at the single site were included. The mean age was 56.2 years (SD 16.0), with $64 \%$ female (Fig. 1 and Table 1). Almost all (98\%) were on subcutaneous methotrexate, and $2 \%$ on oral methotrexate. Mean symptom duration at first visit was 159 days (SD 106), and $83 \%$ met 2010 ACR/ EULAR criteria for rheumatoid arthritis. More than half (59 \%) were rheumatoid factor positive. Majority (57\%) were ever-smokers, $85 \%$ Caucasian, $61 \%$ married, and $56 \%$ had post-secondary education. Half were employed, and $54 \%$ had an income between $\$ 20,000$ and $\$ 100,000$. The mean number of concomitant comorbidities was 3.0 (SD 2.4). There were no statistically significant differences in patient demographics between those who attended early follow-up (103) and those who did not
(37). Anti-CCP was not collected consistently due to lack of universal funding coverage for test, and thus is not reported.

\section{Remission and low disease-activity state}

The proportion of patients in newly achieving either remission or low disease-activity state as per CDAI, SDAI and DAS28 scores are shown in Table 2. At baseline, $66 \%$ of patients were classified as CDAI high disease activity state, with $9 \%$ in LDA state. By 6 weeks, $17 \%$ had achieved CDAI remission with an additional $1 \%$ achieving CDAI remission by 12 weeks; there were a larger proportion of patients entering into early CDAI remission (16\%) versus later remission $(1 \%)(p<0.0002)$. Similar results were found for DAS28 remission (Table 2). By 6 weeks, $59 \%$ had achieved either DAS28 remission or low disease activity state, with $74 \%$

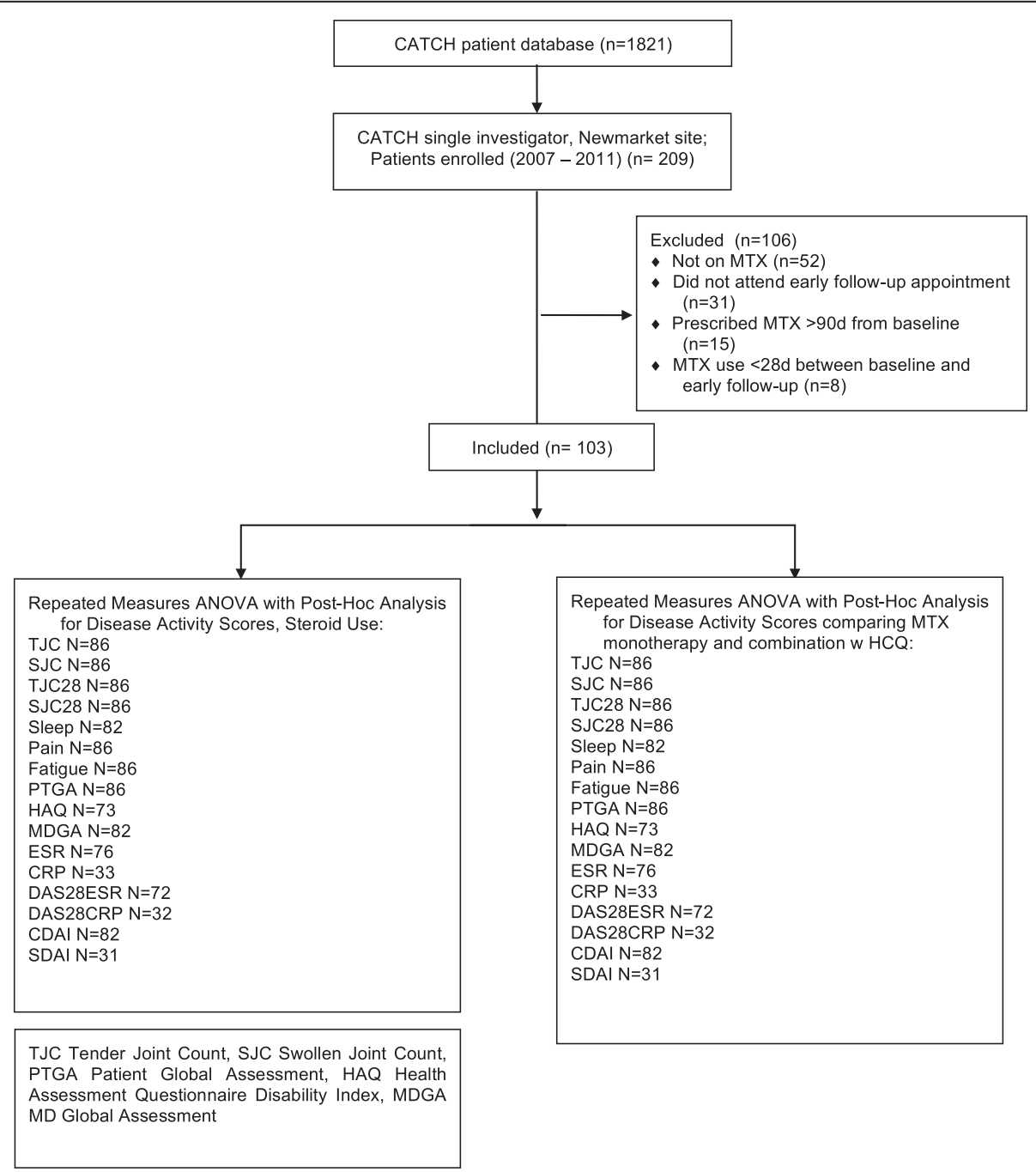

Fig. 1 Patient disposition 
Table 1 Patient demographics of entire cohort, those with 6-week follow-up and those without

\begin{tabular}{|c|c|c|c|c|}
\hline \multirow[t]{2}{*}{ Variables } & All Patients & 6 week follow up visit & No 6 week follow up visit & $p$ value \\
\hline & \multicolumn{4}{|l|}{ Mean Values (SD) } \\
\hline N & 140 & 103 & 37 & \\
\hline Female, no. (\%) & $86(61.4)$ & $64(62.1)$ & $22(59.5)$ & 0.96 \\
\hline Age, mean (SD) (years) & $55.78(15.69)$ & $56.23(15.98)$ & $54.54(13.00)$ & 0.86 \\
\hline Meets 2010 ACR/EULAR Criteria, no. (\%) & $113(80.7)$ & $85(82.5)$ & $28(75.7)$ & 0.90 \\
\hline Mean symptom duration (days) at first visit, mean (SD) & $160.24(107.37)$ & $158.76(105.48)$ & $164.35(114.56)$ & 0.96 \\
\hline Rheumatoid Factor positive, no. (\%) & $156(58.6)$ & $58(58.6)$ & $20(58.8)$ & 0.77 \\
\hline \multicolumn{5}{|l|}{ Smoking Status, no. (\%) } \\
\hline Current smoker & 19 (13.6) & $14(13.6)$ & $5(13.5)$ & \multirow[t]{3}{*}{0.70} \\
\hline Ex-smoker & $64(45.7)$ & $45(43.7)$ & $19(51.4)$ & \\
\hline Never & $57(40.7)$ & $44(42.7)$ & $13(35.1)$ & \\
\hline Caucasian, no. (\%) & $117(83.6)$ & $87(84.5)$ & $30(81.1)$ & 0.85 \\
\hline \multicolumn{5}{|l|}{ Marital status, no. (\%) } \\
\hline Single & $22(15.7)$ & $18(17.5)$ & $4(10.8)$ & \multirow[t]{3}{*}{1.00} \\
\hline Common Law & $6(4.3)$ & $5(4.9)$ & $1(2.7)$ & \\
\hline Married & $91(65.0)$ & $63(61.2)$ & $28(75.7)$ & \\
\hline \multicolumn{5}{|l|}{ Education, no. (\%) } \\
\hline Elementary school & $6(4.3)$ & $5(4.9)$ & $1(2.7)$ & \multirow[t]{6}{*}{0.92} \\
\hline High school & $56(40.0)$ & $40(38.8)$ & $16(43.2)$ & \\
\hline College/Trade school & $41(29.3)$ & $27(26.2)$ & $14(37.8)$ & \\
\hline University Bachelor & $25(17.9)$ & $22(21.4)$ & $3(8.1)$ & \\
\hline Masters & $7(5.0)$ & $6(5.8)$ & $1(2.7)$ & \\
\hline $\mathrm{PhD}$ & $5(3.6)$ & $3(2.9)$ & $2(5.4)$ & \\
\hline \multicolumn{5}{|l|}{ Income, no. (\%) } \\
\hline None & $6(4.3)$ & $5(4.9)$ & $1(2.7)$ & \multirow[t]{6}{*}{0.80} \\
\hline$<\$ 20,000$ & $21(15.0)$ & $17(16.5)$ & $4(10.8)$ & \\
\hline$\$ 20,000-\$ 50,000$ & $46(32.9)$ & $28(27.2)$ & $18(48.6)$ & \\
\hline$\$ 50,000-\$ 100,000$ & $36(25.7)$ & $28(27.2)$ & $8(21.6)$ & \\
\hline$>\$ 100,000$ & $10(7.1)$ & $8(7.8)$ & $2(5.4)$ & \\
\hline Do Not Wish to Answer & $21(15.0)$ & $17(16.5)$ & $4(10.8)$ & \\
\hline \multicolumn{5}{|l|}{ Employment, no. (\%) } \\
\hline Employed & $69(49.3)$ & $51(49.5)$ & $18(48.6)$ & \multirow[t]{8}{*}{0.66} \\
\hline Retired & $43(30.7)$ & $31(30.1)$ & $12(32.4)$ & \\
\hline Homemaker & $10(7.1)$ & $6(5.8)$ & $4(10.8)$ & \\
\hline Student & $6(4.3)$ & $5(4.9)$ & $1(2.7)$ & \\
\hline Disabled & $4(2.9)$ & $2(1.9)$ & $2(5.4)$ & \\
\hline Sick Leave & $3(2.1)$ & $3(2.9)$ & $0(0)$ & \\
\hline Maternity Leave & $1(0.7)$ & $1(1.0)$ & $0(0)$ & \\
\hline Unemployed & $4(2.9)$ & $4(3.9)$ & $0(0)$ & \\
\hline Number comorbidities present, mean (SD) & $3.03(2.32)$ & $2.99(2.38)$ & $3.13(2.21)$ & 0.95 \\
\hline
\end{tabular}

achieving either state by 12 weeks. There were a larger proportion of patients that achieved DAS28 remission in the early vs. late periods $(p<0.00002)$.
Disease activity at early follow-up

Mean change in disease activity scores at each appointment are presented in Additional file 1. Every disease 
Table 2 The proportion of patients in CDAl/SDAI/DAS28 remission, low activity, moderate activity and high activity states at 0, 6 and 12 weeks. CDAl: Clinical Disease Activity Index; SDAl: Simplified Disease Activity Index; DAS28: Disease Activity Score in 28 joints (using ESR)

\begin{tabular}{|c|c|c|c|c|c|}
\hline \multicolumn{2}{|c|}{ Remission score } & \multirow{2}{*}{$\begin{array}{l}\text { Baseline No. (\%) } \\
102\end{array}$} & \multirow{2}{*}{$\begin{array}{l}6 \text { weeks No. (\%) } \\
96\end{array}$} & \multirow{2}{*}{$\begin{array}{l}12 \text { weeks No. (\%) } \\
89\end{array}$} & \multirow{2}{*}{$\begin{array}{l}\text { Early }(0-6 \text { weeks) vs. } \\
\text { Late }(6-12 \text { weeks) }(p)\end{array}$} \\
\hline CDAl & $\mathrm{N}$ & & & & \\
\hline & Remission ( $\leq 2.8$ ) & $0(0.0)$ & $16(16.7)$ & $17(19.1)$ & 0.0002 \\
\hline & Low Activity (2.9-10.0) & $9(8.8)$ & $25(26.0)$ & $48(53.9)$ & 0.15 \\
\hline & Moderate Activity (10.1-22.0) & $26(25.5)$ & $37(38.5)$ & $21(23.6)$ & \\
\hline & High Activity (> 22) & $67(65.7)$ & $18(18.8)$ & $3(3.4)$ & \\
\hline \multirow[t]{5}{*}{ SDAl } & $\mathrm{N}$ & 97 & 58 & 37 & \\
\hline & Remission ( $\leq 3.3$ ) & $0(0.0)$ & $4(6.9)$ & $3(8.1)$ & \\
\hline & Low Activity (3.4-11.0) & $3(3.1)$ & $13(22.4)$ & $16(43.2)$ & 0.08 \\
\hline & Moderate Activity (11.1-26) & $15(15.5)$ & $19(32.8)$ & $13(35.1)$ & \\
\hline & High Activity ( $\geq 26$ ) & 79 (81.4) & $22(37.9)$ & $5(13.5)$ & \\
\hline \multirow[t]{5}{*}{ DAS28 } & $\mathrm{N}$ & 94 & 85 & 76 & \\
\hline & Remission ( $\leq 2.4$ ) & $2(2.1)$ & $22(25.9)$ & $23(30.3)$ & 0.00002 \\
\hline & Low Activity (2.5-3.6) & $9(9.6)$ & $28(32.9)$ & $33(43.4)$ & 0.41 \\
\hline & Moderate Activity (3.7-5.5) & $40(42.6)$ & $29(34.1)$ & $19(25.0)$ & \\
\hline & High Activity ( $\geq 5.5$ ) & $43(45.7)$ & $6(7.1)$ & $1(1.3)$ & \\
\hline
\end{tabular}

activity score differed across time points. Between baseline and 6 weeks, there was a significant difference in mean score for all measures except ESR. Between 6 and 12 weeks, there was a significant difference for all measures except physician global assessment $(p=0.052)$, CRP $(p=0.37)$, DAS28ESR $(p=0.292)$, and SDAI $(p=0.17)$. Between baseline and 12 weeks, there was a significant change in mean scores for all outcomes. Compared to the later period (6-12 weeks), there was a significantly greater early improvement for SJC, SJC28, pain, fatigue, sleep, patient global assessment, physician global assessment, CRP, DAS28ESR, DAS28CRP, CDAI and SDAI.

\section{Methotrexate monotherapy vs. combination DMARD therapy}

Of 103 patients included, 94 patients were on methotrexate monotherapy and only 9 patients were on combination therapy with hydroxychloroquine. Within monotherapy or combination therapy groups, all disease activity measures significantly differed at each time point ( $\mathrm{p} \leq 0.001$ for TJC, SJC, TJC28, SJC, pain, fatigue, sleep, patient global assessment, HAQ, physician global assessment, CRP, DAS28ESR, DAS28CRP, CDAI, SDAI; $p<0.02$ for ESR). Patients treated with MTX monotherapy demonstrated a significantly greater early improvement for SJC, SJC28, pain, fatigue, sleep, PTGA, HAQ, MDGA, DAS28ESR, DAS28CRP, CDAI, and SDAI (all $\mathrm{p} \leq 0.001)$. In the small cohort of patients on combination with HCQ $(n=9)$, there was a non-significant trend toward greater early improvement.

\section{Concomitant steroid use}

Of the 103 patients included, 22 patients did not receive steroids, 15 received oral steroids, 34 received intraarticular steroids only, 13 received intra-muscular steroids only, and 19 patients received combination steroids of at least two routes. Mean disease activity scores at each time point did not significantly differ between steroid groups. The patient group treated with intra-articular steroids alone yielded the highest number of disease measures that demonstrated significant early improvement. Within the small cohort treated with combination steroids $(n=19)$ there was significant early improvement for only two disease measures- fatigue $(p<0.001)$ and PTGA $(p<0.001)$ but this group was likely underpowered. In the cohort not given steroids, there was significant early improvement for TJC and PTGA $(p<0.03)$, as well as MDGA, DAS28ESR and CDAI (all $p<0.02$ ).

\section{Side effects and tolerability}

There were no dropouts secondary to methotrexate intolerance over the first 3 months. Few reported headache (3\%), nausea (4\%), and gastrointestinal symptoms (1\%).

\section{Discussion}

Our study demonstrated an early effect of optimal dosing of scMTX in patients with early RA, with significantly larger early improvement in several disease activity scores and higher proportion achieving early CDAI and DAS28 remission.. Use of concomitant intraarticular steroid yielded the highest number of disease 
activity scores with significant early improvement. The small cohort of patients on combination MTX and HCQ trended toward a greater degree of early improvement, however statistical significance was not achieved - likely secondary to small sample size. Unlike some practices, we often initiate optimal dosing of MTX at the onset of treatment instead of escalating over time. This could contribute to the rapid kinetics of MTX.

Previous data published on MTX therapy in patients with early rheumatoid arthritis have typically used 12 weeks as the initial follow-up point [7]. In this study, the kinetics of sc MTX are more rapid. Many of the patients received concomitant steroids which may facilitate a rapid response, but do not necessarily contribute to the sustained response. Although patients receiving either oral, intramuscular or intra-articular steroids had a higher number of disease activity measures that demonstrated significant early improvement, the cohort of patients who did not receive steroids still demonstrated significant early improvement for five disease activity measures.

Although all patients were from a single centre, baseline characteristics were similar for patients who had 6 weeks visit versus those who did not. This site had baseline demographic data that were not different from the overall CATCH cohort [2]. Assessing patients from a single centre allowed for consistent education, disease scoring and follow-up protocols. There were few patients lost to follow-up and little missing data, avoiding selection bias.

The use of combination therapy with HCQ was also assessed as another potential confounder. Patients who initially received combination therapy had significantly higher disease activity scores at each time point, as compared to those receiving MTX monotherapy, suggesting that patients in the combination group had more active RA at presentation. No disease activity measures within the combination MTX + HCQ group demonstrated a more significant improvement in the early period compared to the later period, likely due to the small N. We could not study oral MTX as it was too rarely used in this practice and there was no triple therapy. The lack of a control group is problematic for the interpretation of the results (po MTX), lack of randomization and prescribing biases of steroid use. The relatively low DAS28 in the ERA incident cohort at onset would tend to give less of a change in DAS28 compared to starting at higher disease activity. Despite these limitations, there is no doubt that even when stratifying by steroid use, there is an early rapid response in those starting optimal doses of sc MTX. A sub-analysis from the TEAR trial showed that even with MTX monotherapy or triple therapy, response at 12 weeks was predictive of future low disease activity, but this study did not look at 6 weeks response
[16]. Certainly MTX seems to have a larger effect early (first 6 weeks) compared to the $2^{\text {nd }} 6$ weeks so perhaps future response can be predicted even earlier with optimal dosing of sc MTX.

\section{Conclusions}

We found that optimal initial dosing with sc MTX in patients with early RA was well-tolerated and yielded rapid (within 6 weeks) significant improvement in many disease measures, including achieving remission and low disease activity state. The use of intra-articular steroids enhanced rapid clinical improvement, as compared to use of steroids via other routes. Future multicenter studies are needed to further assess the rapid kinetics of MTX therapy.

\section{Additional file}

Additional file 1: Comparison of mean change in outcome measurement scores between early period (0-6 weeks) and late period (6-12 weeks). DAS28ESR: Disease Activity Score in 28 joints (using ESR); TJC28: Tender Joint Count in 28 joints; SJC28: Swollen Joint Count in 28 joints; PTGA: Patient Global Assessment; HAQ: Health Assessment Questionnaire Disability Index; MDGA: Physician Global Assessment; ESR: Erythrocyte Sedimentation Rate; CRP: C-Reactive Protein; DAS28CRP: Disease Activity Score in 28 joints (using CRP); CDAl: Clinical Disease Activity Index; SDAl: Simplified Disease Activity Index. (PDF $84 \mathrm{~kb}$ )

\section{Acknowledgements \\ We would like to thank Dr. Vivian P. Bykerk, of the CATCH cohort for allowing Dr. Carter Thorne to extract his single site data.}

\section{Funding}

This is a single site study using some of the CATCH data. The CATCH study was designed and implemented by the investigators and financially supported initially by Amgen Canada Inc. and Pfizer Canada Inc. via an unrestricted research grant since inception of CATCH. As of 2011, further support was provided by Hoffmann-La Roche Ltd., United Chemicals of Belgium (UCB) Canada Inc., Bristol-Myers Squibb Canada Co., Abbott Laboratories Ltd., and Janssen Biotech Inc. (a wholly owned subsidiary of Johnson \& Johnson Inc.). Apart from CATCH, additional support was obtained through a research grant from Medexus Inc.; there was no involvement in data extraction, results or interpretation.

\section{Availability of data and materials}

Data supporting our findings can be found in Additional file 1, which shows the mean change in outcome scores between the early and late time periods (0-6 weeks vs. $6-12$ weeks).

\section{Authors' contributions}

$\mathrm{AO}$ carried out analysis and interpretation of data, drafting the manuscript, revisions of the manuscript and final manuscript approval. CT was involved with conception and design of the study, acquisition of data, assisted with analysis and interpretation of data, revision of the manuscript and final manuscript approval. HK was responsible for acquisition of data, analysis of data, revising manuscript, and final manuscript approval. DT assisted with data collection, revision of the manuscript and final manuscript approval. JP was involved in conception and design, acquisition of data, analysis and interpretation of data, drafting the manuscript, revisions of manuscript, and final approval.

\section{Competing interests}

Carter Thorne: research grant from Medexus; $\mathrm{AO}^{\prime} \mathrm{C}$ Nothing to declare; DT Nothing to declare; HK Nothing to declare; JP Nothing to declare. 


\section{Consent for publication}

Not applicable.

\section{Ethics approval and consent to participate}

All patients signed informed consent and the CATCH project was approved at all sites by the ethics committees (Institutional Review Boards).

\section{Author details}

'Schulich School of Medicine \& Dentistry, University of Western Ontario, 268 Grosvenor St, London, ON N6A 4V2, Canada. ${ }^{2}$ Southlake Regional Health Centre, Newmarket, Canada. ${ }^{3}$ University of Toronto, Toronto, ON, Canada.

${ }^{4}$ Rheumatology, St. Joseph's Health Care, London, ON, Canada.

Received: 13 May 2016 Accepted: 10 August 2016

Published online: 24 August 2016

\section{References}

1. Alamanos Y, Drosos AA. Epidemiology of adult rheumatoid arthritis. Autoimmun Rev. 2005:4(3):130-6.

2. Harris JA, Bykerk VP, Hitchon CA, Keystone EC, Thorne JC, Boire G, Haraou B, Hazlewood G, Bonner AJ, Pope JE. Determining best practices in early rheumatoid arthritis by comparing differences in treatment at sites in the Canadian Early Arthritis Cohort. J Rheumatol. 2013;40(11):1823-30.

3. Jenkins JK, Hardy KJ, McMurray RW. The pathogenesis of rheumatoid arthritis: a guide to therapy. Am J Med Sci. 2002;323(4):171-80.

4. Cush JJ. Early rheumatoid arthritis - is there a window of opportunity? J Rheumatol Suppl. 2007:80:1-7.

5. Haraoui B, Pope J. Treatment of early rheumatoid arthritis: concepts in management. Semin Arthritis Rheum. 2011;40(5):371-88.

6. Nell VP, Machold KP, Eberl G, Stamm TA, Uffmann M, Smolen JS. Benefit of very early referral and very early therapy with disease-modifying antirheumatic drugs in patients with early rheumatoid arthritis. Rheumatology (Oxford). 2004:43(7):906-14

7. Bykerk VP, Keystone EC, Kuriya B, Larche M, Thorne JC, Haraoui B. Achieving remission in clinical practice: lessons from clinical trial data. Clin Exp Rheumatol. 2013;31(4):621-32.

8. Khan ZA, Tripathi R, Mishra B. Methotrexate: a detailed review on drug delivery and clinical aspects. Expert Opin Drug Deliv. 2012;9(2):151-69.

9. Lopez-Olivo MA, Siddhanamatha HR, Shea B, Tugwell P, Wells GA, SuarezAlmazor ME. Methotrexate for treating rheumatoid arthritis. Cochrane Database Syst Rev. 2014;6:CD000957.

10. Pincus T, Yazici Y, Sokka T, Aletaha D, Smolen JS. Methotrexate as the "anchor drug" for the treatment of early rheumatoid arthritis. Clin Exp Rheumatol. 2003;21(5 Suppl 31):S179-185.

11. Yazici Y, Bata Y. Parenteral methotrexate for the treatment of rheumatoid arthritis. Bull Hosp Jt Dis (2013). 2013;71 Suppl 1:46-8.

12. Braun J, Kastner $P$, Flaxenberg $P$, Wahrisch J, Hanke P, Demary W, von Hinuber U, Rockwitz K, Heitz W, Pichlmeier U, et al. Comparison of the clinical efficacy and safety of subcutaneous versus oral administration of methotrexate in patients with active rheumatoid arthritis: results of a six-month, multicenter, randomized, double-blind, controlled, phase IV trial. Arthritis Rheum. 2008;58(1):73-81.

13. Hazlewood GS, Thorne JC, Pope JE, Lin D, Tin D, Boire G, Haraoui B, Hitchon CA, Keystone EC, Jamal S, Bykerk VP, CATCH Investigators. The comparative effectiveness of oral versus subcutaneous methotrexate for the treatment of early rheumatoid arthritis. Ann Rheum Dis. 2016;(6):1003-8. doi:10.1136/annrheumdis-2014-206504.

14. Weinblatt ME. Toxicity of low dose methotrexate in rheumatoid arthritis J Rheumatol Suppl. 1985;12 Suppl 12:35-9.

15. Wevers-de Boer K, Visser K, Heimans L, Ronday HK, Molenaar E, Groenendael $J$ H, Peeters AJ, Westedt ML, Collee G, de Sonnaville PB, et al. Remission induction therapy with methotrexate and prednisone in patients with early rheumatoid and undifferentiated arthritis (the IMPROVED study). Ann Rheum Dis. 2012;71(9):1472-7.

16. Curtis JR, McVie T, Mikuls TR, Reynolds RJ, Navarro-Millan I, O’Dell J, Moreland LW, Bridges Jr SR, et al. Clinical response within 12 weeks as a predictor of future low disease activity in patients with early RA: results from the TEAR trial. J Rheumatol. 2013;40:572-8.

\section{Submit your next manuscript to BioMed Central and we will help you at every step:}

- We accept pre-submission inquiries

- Our selector tool helps you to find the most relevant journal

- We provide round the clock customer support

- Convenient online submission

- Thorough peer review

- Inclusion in PubMed and all major indexing services

- Maximum visibility for your research

Submit your manuscript at www.biomedcentral.com/submit 